\title{
New Identified Anthocyanins from Sudanese Roselle: Potential Candidates for inhibition of Xanthine Oxidase
}

\section{Arwa El-Naeem}

University of Khartoum

Sahar Abdalla ( $\nabla$ sahar.abdalla@uofk.edu )

University of Khartoum

Ibrahim Ahmed

University of Khartoum

\section{Research Article}

Keywords: anthocyanin pigments, Sudanese roselle, inhibition of xanthine oxidase (XO)

Posted Date: April 2nd, 2021

DOI: https://doi.org/10.21203/rs.3.rs-370476/v1

License: (1) (1) This work is licensed under a Creative Commons Attribution 4.0 International License.

Read Full License 


\section{Abstract}

This study aims to identify anthocyanin pigments in Sudanese roselle and examine their inhibitory activity toward xanthine oxidase (XO) enzyme via in silico docking approach. A number of four samples of Sudanese roselle (red and white) from different regions of Sudan were investigated by high sensitive technique, i.e. LC-MS to identify anthocyanins. Four anthocyanins were identified in all samples; delphinidin-3-glucoside (Dp-3-glu), cyanidin-3-sambubioside (Cy-3-sam), pelargonidin chloride (Pg Chloride), and petuinidin-3-glucoside (Pt-3-glu); in addition to one flavanol; gossypetin (Goss). The anthocyanins of the white samples are suggested to be presented in the yellowish or colorless pseudo base structures. The identified anthocyanins were tested against the inhibition toward xanthine oxidase via molecular docking. All anthocyanins were found to be excellent XO inhibitors superior to the most recent commercially used hyperuricemia drug; i.e. topiroxostat. The binding energies of the complexes (ligand-XO) are lower than the energy of the topiroxostat-XO complex. The binding energies order is: $\mathrm{pt}-3$ dp-3-glu > cy-3-sam > goss > pg chloride. According to our investigation, roselle anthocyanins are considered as good potential future $\mathrm{XO}$-inhibitors drugs; and promising candidates to treat several related diseases.

\section{Introduction}

Anthocyanins, are water-soluble plant pigments found in almost all members of the plant kingdom providing colors for most of plant tissues (leaves, stems, roots, flowers and fruits) [1-3]. Rich anthocyanins sources used as potential edible colorants involve colored fruits such as berries, cherries, and many dark-colored vegetables such as black currant, red onion, red radish as well as Hibiscus calyces [1].

Anthocyanins have different types according to composition, and chemical structures. These types and their concentration are varying not only between different species, but also among the fruits [4]. The structure of anthocyanins have basic common skeleton composed of anthocyanidin and glycoside. The anthocyanidin (aglycone) is a polyhydroxy or a polymethoxy derivative of 2-phenyl-benzopyryliurn or flavylium salt $[1,2]$. The common structure of anthocyanins is illustrated in Fig. 1.

There are six major anthocyanidins, mentioned in the caption of Fig. 1, to which $90 \%$ of all identified anthocyanins based on i.e. cyanidin, delphinidin, pelargonidin, peonidin, petudinin, and malvidin [1-3].

Hibiscus sabdariffa L., Roselle - or Karkade, is an annual [4], or perennial herb or sub-shrub [5], grows in tropical and subtropical regions [4-6]. Sudan is one of its largest exporters in the world. It grows there in various parts particularly the western $[4,6,7]$.

In many countries roselle is considered as traditional medicinal in the treatment of many diseases like; relieving pain and coughs also treating liver disease, fever hypercholesterolemia, hypertension, antispasmodic and antimicrobial agent etc ... [10]. The calyx containing the attractive entities of the plant that responsible of its color, flavor and aroma $[4,6,8]$, however, other plant parts are also used $[4,7]$, 
The anthocyanins pigment can be used as natural edible dye in industries [9]. Anthocyanins-rich foods in general are recommended to use by nutritionists due to their antioxidants effects and ability to inhibit some enzymes for instance; xanthine oxidase, lipoxygenases, and nicotine amide adenine dinucleotide phosphate (NADPH) oxidase $[1,11]$. Roselle extracts are used to the treatment of hyperlipidemia, inflammatory diseases, cancer, and reduction of the incidence of liver lesions [12]. Anthocyanins plant extracts are always mixtures of different anthocyanins and colorless flavonoids [13]. The red color of Roselle calyces was attributed to the presence of dephinidin-3-sambubioside, cyanidin-3-sambubioside, delphinidin-3-glucoside and cyanidin-3-glucoside anthocyanins $[6,7,14]$. A study on Sudanese Roselle determined delphinidin-3-sambubioside to be the major anthocyanin responsible for the reddish violet color [6].

The characterization of anthocyanins is usually done by the chromatographic spectrophotometric and capillary electrophoresis (CE) methods [3]. Recently; the most common used technique for characterization of anthocyanins is high performance liquid chromatography (HPLC), usually hyphenated with mass spectrometry (LC-MS) [3].

Nowadays modern techniques allow identification of several anthocyanin types. However; nonavailability and high cost of pure anthocyanin standards present a problem specifically when using UV/Vis spectroscopy or HPLC techniques [3].

Recently, modern techniques are unitized to identify pelargonidin chloride, pelargonidin-3-glucoside and petunidin-3-glucoside as well as other anthocyanins from Sudan Roselle [14, 15].

The ability of anthocyanins to inhibit xanthine oxidase $(X 0)[1,11]$ withdrew our attention to the use of Roselle as traditional medicinal drink to reduce the human blood pressure in Sudan. Xanthine oxidase is a highly versatile enzyme, widely distributed within the different kingdom animalea species [16]. It catalyzes the hydroxylation of hypoxanthine to xanthine and xanthine to uric acid, accompanying with the production of; reactive oxygen species ROS $[16,17]$. The increase of the activity of the enzyme leads to the accumulation of uric acid and ROS leading to the infection by several diseases, mainly hyperuraecimia and gout with other associated chronic diseases such as; hypertension, metabolic syndrome, cardiovascular diseases, diabetes, obesity, cancer and hyperlipidemia $[16.17,18]$.

Enzyme inhibition will be carried out herein via docking study. Any pharmacologically active compound acts through interaction with targets like proteins and nucleic acids [19]. The most recent drug to treat the hyperuricemia is topiroxostat [20].

The objective of the current research is to identify anthocyanins in two different Sudan Roselle samples i.e., red and white, special attention paid to study the ability of the anthocyanins to inhibit xanthine oxidase enzyme and explore most promising drug related to different diseases. In particular, the binding energies obtained from our identified anthocyanins will be compared with that obtained from topiroxostat. 


\section{Results}

\section{LC-MS/MS Identification of Anthocyanins:}

From the samples we have characterized four different types of anthocyanins in addition to one falvonol. Table.1 represents the LC-Q-TOF-MS identification of anthocyanins and falvonol gossypetin.

Identification was accomplished according to the chemical formulas and molecular weights as matching the instrument library using Mass Lynx 4.1 software. Our findings were compared to the theoretical values of the corresponding expected anthocyanins from Pubchem [21].

\section{Molecular Docking:}

According to our docking results; low binding energies of roselle anthocyanins complexes with xanthine oxidase enzyme were obtained. The binding energies of the (anthocyanin- $\mathrm{XO}$ ) and (topiroxostat- $\mathrm{XO}$ ) complexes are reported in Table. 2.

\section{Discussion}

The anthocyanins cy-3-sam, and dp-3-glu were identified in all samples. This is in agreement with many previous studies considering Sudan $[14,15]$ and non Sudan Roselle types $[10,22,23]$

The presence of dp-3- glu anthocyanin in our samples is in accordance with that obtained by Obouayeba et al. [24] in his investigation of Abdjanian Roselle. Both Pg chloride, and pt-3-glu anthocyanins characterized herein are recently identified among other types of anthocyanin from Sudan Roselle analyzed by UHPLC-ESI-MS/MS [15], which were not identified in other studies using Abdjanian Roselle ref. $[22,24]$. This may be due to the geographical difference.

The identification of new anthocyanins in our samples confirms the validity of the LC-MS being the most powerful and suitable technique for analyzing anthocyanins, as; it provides both separation and the finger print identification through MS. The preferred solvent, by convention; for anthocyanins extraction is methanol in accordance with results reported by Wrolstad et al. [25].

According to our investigation, we have found that the white types of Roselle have the same anthocyanins that found in the red. This can be explained by the presence of different equilibrium structures of anthocyanins with a dominant one depend on the plant physiology and climate condition. Therefore, it can be suggested that; the anthocyanins of the white roselle types; are presented in their yellowish or colorless pseudo base structures and this finding supports the results obtained therein [3].

The identification of anthocyanins was accompanied by identification of a flavanol i.e., goss, in agreement with Harborne [13]. The existence of flavanol in our samples is in consistent with previous result obtained in Alamin's PhD thesis [14] and can be directly attributed to the similarity in the origin of the plant and modern technique of analysis used i.e., LC-MS. 
The presence and absence of one or more anthocyanin in roselle from Sudan compared to other countries can be attributed to soil and climatic conditions that distinguishing various zone in accordance with former studies $[14,22]$.

The obtained anthocyanins and the flavonol were tested against inhibiting XO. All used ligands exhibited low binding energies, therefore; strong ligand-enzyme complexes resulted. These binding energies, even the highest one obtained by pg chloride, are less than the binding energy obtained by standard drug, c.a.-8.6 kcal $/ \mathrm{mol}$. The order of decreasing energy of anthocyanins is: pg chloride $<$ goss $<$ pt-3-glu $<$ dp-3glu < cy-3-sam.

The slight differences in the binding energy values among ligands depended on the anthocyanin type i.e., differences in anthocyanin structures (particularly in the attached sugar moiety and in the type and position of the substituent groups). This is in agreement with previous study [1] that; anthocyanins with different chemical structures have different biological properties ensuring optimal molecular fitting into binding sites.

Obtaining low binding energies in all ligands may attribute to glucoside moiety and the free hydroxyl groups. Due to; being the most important moieties shared in binding interactions. This result is in agreement with results reported by Wallace et al. [11]. Both pg chloride and flavonol goss are less active ligands due to the lack glucoside moieties.

Structures of the identified anthocyanins (see Fig. 1), represents the absence of a hydroxyl group at C-3 position, a character responsible for slightly enhancing the inhibition effect of $\mathrm{XO}$ according to Kostić and his co-workers [17].

The flavonol goss in contrast to anthocyanins, contains hydroxyl groups at C-5 and C-7 and a double bond between $\mathrm{C}-2$ and $\mathrm{C}-3$. These are essential for a high inhibitory activity toward XO [17]. Thus; being superior in its activity compared to pg chloride.

Different intermolecular bond types were determined as visualizing our results including $H$-bonds, in addition to; hydrophobic ( $\pi$-alkyl, $\pi$-sigma and Alkyl) and electrostatic ( $\pi$-cation) bond types, in agreement with study reported previously [16]. The intermolecular binding interactions of each of the ligand (anthocyanins, flavonol, and the standard drug) with the FAD active site of XO are illustrated in Fig. 3. The shortest intermolecular $\mathrm{H}$-bond distances were less than the sum of the Van der Waals radii of atoms [26].

Since hyperuraecimia and gout are known to be associated to hypertension and other several diseases, our docking results strongly supporting the local use of roselle anthocyanins in folk medicine for hypertension treatment. Consequently; the obtained roselle anthocyanins are potential drug candidates for gout and hypertension treatment.

\section{Experimental}




\section{Samples Preparation:}

Red and white dried Roselle calyces originated from four different sites in Sudan were used. Each sample was firstly selected, cleaned from agricultural residues and ground to fine powder using an electrical grinder (Monolex original France). Then well homogenized, packed in polyethylene bags, labeled from 1 to 4 , and stored at room temperature $\left(20-30{ }^{\circ} \mathrm{C}\right)$ till used.

\section{Reagents:}

All reagents were of HPLC grade: formic acid, acetonitrile, methanol and water. Except $\mathrm{HCl}$ in methanol and chloroform that used in extracts preparation were of analytical grade.

\section{Instruments:}

Rotatory evaporator (Heidolph Laborota 4000 efficient HB digital), UPLC-Q- TOF-MS ( XEVO-G2 QTOF YCA119 Waters, using Acquity UPLC HS T3 $(100 \times 2.1 \mathrm{~mm}, 1.8 \mu \mathrm{m})$ (Waters) column maintained at $45^{\circ} \mathrm{C}$ in a column oven.

\section{Methods:}

\section{Preparation of purified extract:}

Anthocyanins were extracted with $250 \mathrm{ml}$ acidified methanol using soxhlet apparatus [5] at $55^{\circ} \mathrm{C}$ for $6-8$ $\mathrm{h}$ till reaching faint colored extracts. Extracts was concentrated to small volumes using rotatory evaporator (Heidolph Laborota 4000 efficient HB digital) and equal volumes of water were added to each extract in order to achieve possible simple liquid/liquid purification with chloroform of a doubled volume of the total aqueous acidified methanolic extract.

\section{LC-MS/MS Identification:}

Anthocyanins were identified in the purified samples extracts by LC-MS/MS technique in the LC-MS lab, Beijing University of Chemical Technology BUCT, China. The analysis was carried out according to the method described by Cahlíková., et al [15] with some modifications. The conditions of the chromatographic run were as follows:

Gradient mobile phase composed of water acidified with $0.1 \%$ formic acid (solvent $A$ ) and acetonitrile (solvent B). The flow rate and sample injection volume were; $0.4 \mathrm{ml} / \mathrm{min}$ and $3.00 \mu \mathrm{l}$; respectively. 
Initiating by $A$ to $B$ 9:1 for 1 min, then $85 \%$ A, 15\% B 1-5 min, then 5-10 min 40\% A, 60\% B, 100\% acetonitrile with no water 10-13 min. Thereafter the concentration was returned to 90 and $10 \%$ till 13-15 $\min$.

The ion source of Q-TOF detector was set at the positive ESI polarity mode. The optimum conditions of QTOF system which performed in the Resolution Mode that applied for the run were: capillary voltage of $+3.000 \mathrm{kV}$, ion source temperature: $100{ }^{\circ} \mathrm{C}$, extractor: $4.0 \mathrm{~V}$; RF lens: $0.3 \mathrm{~V}$. Mass range m/z: 50 to 2000 Da. Nitrogen as the desolvation gas at a flow rate of $800 \mathrm{l} / \mathrm{h}$ and at a temperature of $400{ }^{\circ} \mathrm{C}$. Nitrogen was used also as the cone gas $(20 \mathrm{l} / \mathrm{h})$, and argon as the collision gas. The cone voltage, collision energy, and well time were carefully optimized for each compound and transition individually (cone voltage set at $4 / 30 \mathrm{~V}$, collision energy set finally at $6 \mathrm{eV}$ ).

The software that used for the MS control and data gathering was Mass Lynx 4.1.

\section{Molecular docking:}

Molecular docking is a method used for testing the ability and screening specific small compounds (ligands) to fit into a previously identified therapeutic target (the receptor; usually protein enzyme) [20,27]. Several docking programs are applied. AutoDock Vina, GOLD, and MOE-Dock are top ranked with best scores [27].

In the present paper, was utilized AutoDock Vina program for the docking of the anthocyanin ligands, to the active site of the xanthine oxidase enzyme after performing the required preparations. First of all; the protein crystal structure and ligands 3D structures were downloaded from RCSB Protein Data Bank (1FIQ PDB code) and Pubchem online databases; respectively $[21,28]$. Then the suitable binding site of the protein, i.e flavin adenine dinucleotide FAD, was obtained using Chimera software [29] and the ligands were converted via Open Babel software [31] from SDF format to pdbqt. Thereafter; using autodock tools (ADT) [30] all water molecules in the protein.pdb file were removed, hydrogen atoms were added, and the grid box dimensions were set, then the file saved as pdbqt. On the other hand ligands were prepared by detecting torsions for each structure and saved as a pdbqt file. (PDBQT format)

The AutoDock Vina order was then created after preparing the configuration files [32]. The process was repeated for each docking trial regarding all ligands. The docked ligands were: Delphinidin-3-glucoside (Dp-3-glu), Cyanidin-3-sambubioside (Cy-3-sam), Pelargonidin chloride (Pg Chd), Petuinidin-3-glucoside (Pt-3-glu), the flavonol Gossypetin (Goss) and Topiroxostat (FYX-051). Their structures are depicted in Fig. 2.

\section{Data analysis}

The Discovery Studio Visualizer [33] and PyMOL Viewer [34] were used to visualize interactions of the binding site amino acid residues with the ligands. The mode with the lowest binding energy was choosed in each (out.pdbqt) file. 


\section{Conclusion}

Four anthocyanins and a flavonol were investigated in this study in four Sudan roselle samples. These can be used as potential drug candidates for the treatment of diseases associated to XO enzyme increased activity. This finding is compatible with previous reported researches that; inhibitors of XO are important for medicate several diseases, such as; diabetes, hypertension and cardiovascular disease.

\section{Declarations}

\section{Author contributions}

All authors formulate research goals and aims.

AE performed sample collection, develop chemical methodology, conducting the research performing the chemical and computational (docking) experiments, analyze results writing the initial draft and writing revisions.

SA and IA performed student supervision, and managed the research.

SA carried out the commentary and revision of writing.

\section{Competing interests}

The author(s) declare no competing interests.

\section{References}

1. Zia-Ul-Haq, M., Riaz, M. \& Saad, B. Anthocyanins and human health: biomolecular and therapeutic aspects(Springer International Publishing, 2016).

2. Hirsch, G. E. \& Martins, L. A. M. Anthocyanins: chemical features, food sources and health benefits in Handbook of Anthocyanins. 227-248(Nova Science Publishers, Inc., 2015).

3. Welch, C., Wu, Q. \& Simon, J. (2008). Recent advances in anthocyanin analysis and characterization. Curr. Anal. Chem. 4, 75-101 (2008).

4. Mohamed, B. B., Sulaiman, A. A. \& Dahab, A. A. Roselle (Hibiscus sabdariffa L.) in Sudan, cultivation and their uses. Bull Env. Pharmacol Life Sci. 1, 48-54 (2012).

5. Okereke, C. N., Iroka, F. C. \& Chukwuma, M. O. Phytochemical analysis and medicinal uses of Hibiscus sabdariffa. Int. J. Herb. Med. 2, 16-19 (2015).

6. AL-Shoosh, W. G. A. A. Chemical composition of some roselle (Hibiscus sabdariffa) genotypes(UNIVERSITY OF KHARTOUM, 1997).

7. Adam, R. I., Gibla, O. A. M. \& Mohammed, E. O. A. Physicochemical analysis of some natural Sudanese juices. Int. J. Multidiscip. Res. Dev. 3, 94-96 (2016). 
8. Abou-Arab, A. A., Abu-Salem, F. M. \& Abou-Arab, E. A. Physico- chemical properties of natural pigments (anthocyanin) extracted from Roselle calyces (Hibiscus subdariffa). J. Am. Sci. 7, 445-456 (2011).

9. Inggrid, H. M., Jaka \& Santoso, H. Natural red dyes extraction on roselle petals. IOP Conf. Ser. Mater. Sci. Eng. 162, 012029 (2016).

10. Riaz, G. \& Chopra, R. A review on phytochemistry and therapeutic uses of Hibiscus sabdariffa L. Biomed. Pharmacother. 102, 575-586 (2018).

11. Wallace, T. C. Anthocyanins in cardiovascular disease Prevention in Handbook of Anthocyanins in Health and Disease. Adv. Nutr. Int. Rev. J. 2, 165-198 (2011).

12. Kong, J. M., Chia, L. S., Goh, N. K., Chia, T. F. \& Brouillard, R. Analysis and biological activities of anthocyanins. Phytochemistry. 64, 923-933 (2003).

13. Harborne, J. B. Phytochemical methods: a guide to modern techniques of plant analysis(Chapman and Hall, 1998).

14. Alamin, Y. K. Physicochemical study of Hibiscus sabdariffa L. (Karkade) genotypes(UNIVERSITY OF KHARTOUM, 2012).

15. Cahlíková, L. et al. Anthocyanins of Hibiscus sabdiffera Calyces from Sudan. Nat. Prod. Commun. 10, 77-79 (2015).

16. Umamaheswari, M. et al. Discovery of potential xanthine oxidase inhibitors using in silico docking studies. 3,240-247(2011).

17. Kostić, D. A. et al. Xanthine oxidase: isolation, assays of activity, and inhibition. J. Chem. 2015, 1-8 (2015).

18. Gliozzi, M., Malara, N., Muscoli, S. \& Mollace, V. The treatment of hyperuricemia. Int. J. Cardiol. 213, 23-27 (2016).

19. Bisht, N. \& Singh, B. K. Role of computer aided drug design in drug development and drug discovery. Int. J. Pharm. Sci. Res. 9, 1405-1415 (2018).

20. Luna, G., Dolzhenko, A. V. \& Mancera, R. L. Inhibitors of xanthine oxidase: scaffold diversity and structure-based drug design. ChemMedChem. 10,1002(2019).

21. Pagadala, N. S., Syed, K. \& Tuszynski, J. Software for molecular docking: a review. Biophys. Rev. 9, 91-102 (2017).

22. Berman et al. The Protein Data Bank. Nucleic Acids Res. 28, 235-242 (2000).

23. Enroth et al. T. and F.Crystal structures of bovine milk xanthine dehydrogenase and xanthine oxidase: structure-based mechanism of conversion. Proc. Natl. Acad. Sci. 97, 10723-10728(2000).

24. Kim, S. et al. PubChem 2019 update: improved access to chemical data. Nucleic Acids Res. 47, 1102-1109 (2019).

25. Pettersen, E. F. et al. E. UCSF Chimera-a visualization system for exploratory research and analysis. J Comput Chem. 25, 1605 - 1512(2004).

26. O'Boyle, N. M. et al. Open Babel: An open chemical toolbox. J. Chem inf. 3, 33 (2011). 
27. Sanner, M. F. \& Python A Programming Language for Software Integration and Development. J. Mol. Graphics Mod. 17, 57-61 (1999).

28. Trott, 0 . \& Olson, A. J. AutoDock Vina: improving the speed and accuracy of docking with a new scoring function, efficient optimization, and multithreading. J. Comput. Chem. 31, 455-461 (2010).

29. Diego, S. \& Dassault Systèmes, B. I. O. V. I. A.Discovery Studio Visulizer, Dassault Systèmes, (2016).

30. Pacôme, O. A. et al. Phytochemical and antioxidant activity of roselle (hibiscus sabdariffa I.) petal extracts. RJPBCS. 5, 1454 (2014).

31. Chumsri, P., Sirichote, A. \& Itharat, A. Studies on the optimum conditions for the extraction and concentration of roselle (Hibiscus sabdariffa Linn.) extract. Songklanakarin J. Sci. Technol. 30, 133139 (2008).

32. Obouayeba, A. P., Okoma, K. M., Kouakou, T. H. \& Diarrassouba. M., Diabaté. S., and Phytochemical Characterisation and Antioxidant Activity of Hibiscus sabdariffa (Malvaceae) Calyx Extracts. JAAS. 3, 39-46 (2015).

33. Wrolstad, R. E. et al. Pigments and Colorants in Handbook of Food Analytical Chemistry. 1-69(John Wiley and Sons, Inc., 2004).

34. Bondi, A. Ñ'an der Waals Volumes and Radii. J. Phys. Chem. 68, 441-451 (1964).

35. The PyMOL Molecular Graphics System, Version 1.2r3pre, Schrödinger, LLC.

\section{Tables}

Table.1 LC-Q-TOF-MS identification.

\begin{tabular}{|l|l|l|}
\hline Measured $m / z$ & Theoretical $m / z$ & Name of the identified compound \\
\hline 465.1033 & 465.387 & Dp-3-glu \\
\hline 581.1506 & 581.503 & Cy-3-sam \\
\hline 306.698 & 306.698 & Pg Chloride \\
\hline 479.414 & 479.414 & Pt-3-glu \\
\hline 318.237 & 318.237 & Goss \\
\hline
\end{tabular}

Table.2 The binding energies of the (anthocyanin-XO) and (topiroxostat-XO) complexes:

\begin{tabular}{|l|l|}
\hline Docked ligand & Binding energy $(\mathrm{kcal} / \mathrm{mol})$ \\
\hline Cy-3-sam & -10.20 \\
\hline Dp-3-glu & -9.50 \\
\hline Pt-3-glu & -9.50 \\
\hline Goss & -9.40 \\
\hline Pg Chloride & -8.80 \\
\hline Topiroxostat & -8.60 \\
\hline
\end{tabular}


Figures

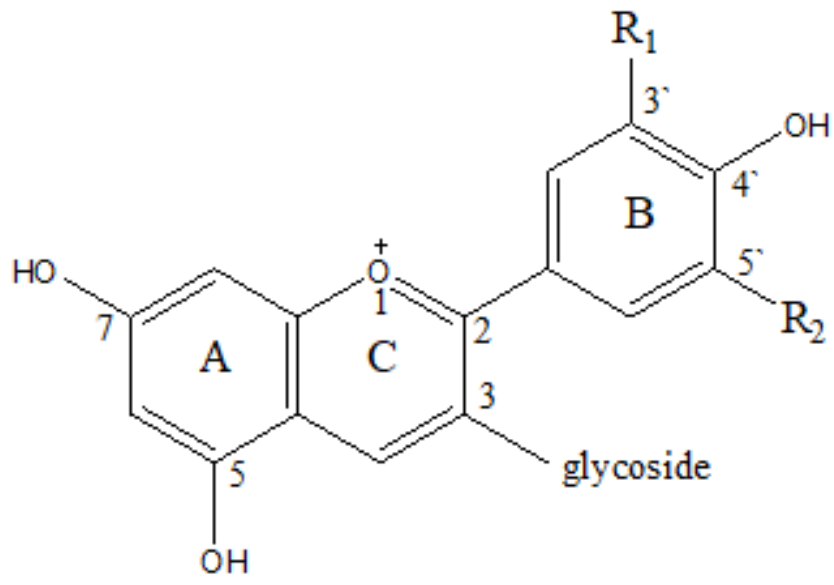

Figure 1

General structure of anthocyanins, Pelargonidin; R1 = H, R2= H, Cyanidin R1= OH, R2 = H, Peonidin; R1 $=\mathrm{OCH} 3, \mathrm{R} 2=\mathrm{H}$, Delphinidin; R1 = OH, R2= OH, Petunidin; R1 = OCH3, R2 = OH and Malvidin; R1 = OCH3, R2 $=\mathrm{OCH} 3$.

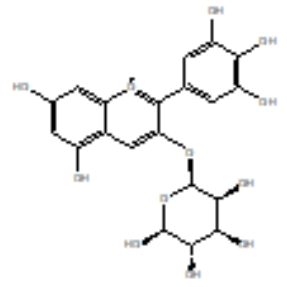

Dp-3-glu<smiles>OC1=C(c2ccc(O)c(O)c2)Oc2c(O)c(O)cc(O)c2C1O</smiles>

Goss

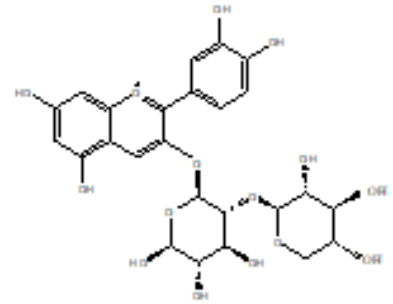

Cy-3-sam<smiles>CCC1CC(C2CC(C)C(C)C(C)C2)C(CC2CC(C)C(C)C(C)C2C)CC1C</smiles>

Pt-3-glu

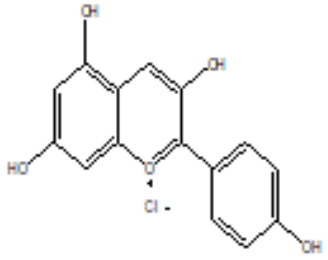

Pg Chloride<smiles>C#Cc1cc(-c2nc(-c3ccccc3)ns2)ccn1</smiles>

FYX-051

Figure 2 
Chemical structures of the ligands used in the molecular docking.
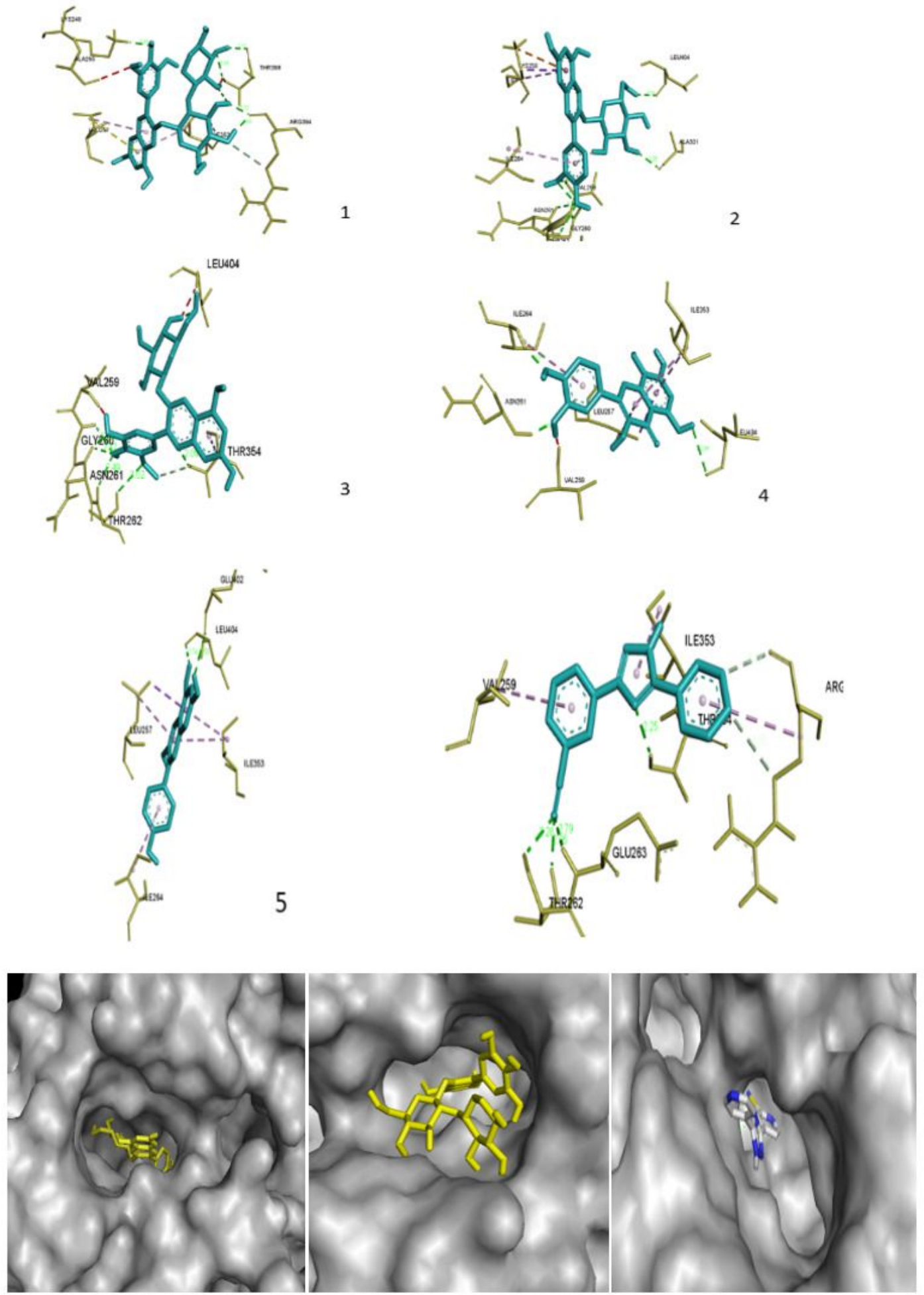

\section{Figure 3}

Top panel: Interaction of ligands with the binding site. (a) Structures numbered from 1 to 5 are XO complexes of cy-3-sam, dp-3-glu, pt-3-glu, goss and pg chloride respectively. The last one is the complex of the standard drug Topiroxostat. The ligands structures are light blue colored and the binding amino acid residues are pale yellow one. The bonds are represented in dashed lines; classical H-bonds are green 
color, $\mathrm{C}-\mathrm{H}$ bond grey colored, $\pi$-sigma dark violet, $\pi$-cation orange, $\pi$-alkyl violet, and Alkyl faint violet. The bonds in red color are non-favor bonds. Bottom panel: (b) Molecular surface view illustrates fitting of ligands at the FAD active site, (1) dp-3-glu. (2) cy-3-sam. (3) topiroxostat. 